\title{
Effect of Electrical Stimulation at Different Time on Mitochondrial Function of C2C12 Myotubes
}

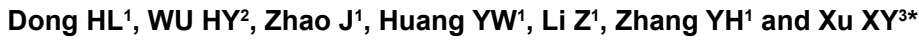 \\ ${ }^{1}$ Sports College, Jinan University, Guangzhou 510632, China \\ ${ }^{2}$ Nanfang Hospital, Southern Medical University, 51000, China \\ ${ }^{3}$ School of Physical Education, South China Normal University, Guangzhou 510631, China
}

\begin{abstract}
Objective: To study the effect of different time of electrical stimulation on mitochondrial function of $\mathrm{C} 2 \mathrm{C} 12$ myotubes, and further explore its molecular mechanism.

Methods: An electrical stimulation was given 7 days after $\mathrm{C} 2 \mathrm{C} 12$ myotubess differentiation, of which intensity was $30 \mathrm{~ms}, 3 \mathrm{~Hz}$, and the stimulation time was $60 \mathrm{mins}, 120 \mathrm{mins}$, and $180 \mathrm{mins}$, respectively. A total of four experimental 4 groups, including control group (Con), 60 mins group (E60), 120 mins (E120) and 180 mins (E180). Microscope was used to observe the muscular myotubes form; Kits were to detect MDA, SOD and ROS; Western blot was used to detect the expression of autophagy proteins and mechanism proteins, including PGC1, p-ULK, SIRT1 and SIRT3; Flow cytometry technology was used to detect muscle mitochondrial membrane potential.
\end{abstract}

Results: There was no significant difference of $\mathrm{C} 2 \mathrm{C} 12$ myotubes form after different electrical stimulation Compared with the control group, E60 had no significant difference of mitochondrial membrane potential $(p>0.05)$; but MDA, ROS, SIRT3 increased significantly $(p<0.05), p-U L K$ and PGC1 increased significantly $(p<0.01)$, SIRT1 decreased significantly $(p<0.05)$. In E120, MDA, ROS, SIRT3 and PGC1 increased significantly $(p<0.01)$, SOD and mitochondrial membrane potential decreased significantly $(p<0.05)$. In E180, MDA and ROS increased significantly $(p<0.01)$, SOD and mitochondrial membrane potential decreased significantly $(p<0.01)$

Conclusion: Moderate electrical stimulation (60 and 120 mins) can significantly activate oxidative stress, and further promote SIRT3, PGC1 and p-ULK expression, and further promote the mitochondrial membrane potential, while excessive stimulation (180 mins) has the opposite effects.

Keywords: Electrical stimulation; C2C12 muscle myotubes; Sirts; Mitochondrial function

\section{Introduction}

In recent years, many researchers have used different exercise types or intensity of exercise programs to explore the phenomenon and mechanism of oxidative stress. Many studies have confirmed that an acute strenuous exercise leads to an imbalance between oxidation and antioxidant system in body, an enhancement in lipid peroxidation and a-significantly increase in MDA level in skeletal muscle. The mitochondrial membrane potential and free calcium ion content were significantly reduced and MDA content was significantly increased in skeletal muscle of rats after two weeks of moderate intensity treadmill exercise [1] Mitochondrial function is one of the most cutting-edge fields in the current research [2]. And the specific mechanism of mitochondrial function deserves further study. Mitochondria, as the center of energy metabolism, not only maintain various physiological processes in resting state, but also play an extremely important role in movement state. Therefore, it is more meaningful to study the role of mitochondrial function in motor body. Other studies have shown that [3-5] p-ulk and sirt $1 / 3$ (silent information regulatory factor 2 related enzymes $1 / 3$ ) have an important regulatory effect on the level of oxidative stress. They can reduce reactive oxygen free radicals and improve the living environment. $\mathrm{C} 2 \mathrm{C} 12$ (mouse $\mathrm{C} 3 \mathrm{H}$ muscle my oblast) cells were used in this study. The differentiated $\mathrm{C} 2 \mathrm{C} 12$ myotubes could complete the same contraction as in vivo skeletal muscle [6], while avoiding the effects of other organs, tissues, cells and hormones in vivo experiments. The effects of the mitochondrial function can be studied in real time with different exercise combination. The research provided a theoretical basis for sports injury and recovery.

\section{Materials and Methods}

\section{Research material}

C2C12 (mouse $\mathrm{C} 3 \mathrm{H}$ muscle my oblast); DMSO, DEPC, L-glutamine (sigma, USA); HEPES (MBCHEM, India); fetal bovine serum, DMEM medium, horse serum (GIBCO, USA); MDA, SOD, ROS kits (Nanjingjiancheng, China); anti-Sirt1, Sirt3, p-ULK (CST, USA); anti-PGC1 (abcam, USA); Tetramethylrhodamine methyl ester, perchlorate (TMRM, Shanghaijieling, China).

\section{Cell culture}

$\mathrm{C} 2 \mathrm{C} 12$ cells were cultured in growth medium containing $4.5 \mathrm{~g} / \mathrm{L}$ glucose, $4 \mathrm{mM}$ glutamine, 10\% FBS, $100 \mathrm{ug} / \mathrm{mL}$ penicillin, and $50 \mathrm{ug} /$ $\mathrm{mL}$ streptomycin at $37^{\circ} \mathrm{C}$ under a $5 \% \mathrm{CO}_{2}$ atmosphere. Cells were then plated on $35 \mathrm{~mm}$ culture dishes at a density of $2 \times 10^{5}$ cells/well in $2 \mathrm{~mL}$ of growth medium. Three days after plating, the cells had reached $90 \%$ $95 \%$ confluence (day 0). Differentiation was induced by incubating cells in differentiation medium containing $4.5 \mathrm{~g} / \mathrm{L}$ glucose, $4 \mathrm{mM}$ Glutamine, $2 \% \mathrm{HS}, 100 \mathrm{ug} / \mathrm{mL}$ penicillin, and $50 \mathrm{ug} / \mathrm{mL}$ streptomycin. The differentiation medium was changed every $24 \mathrm{~h}$.

${ }^{*}$ Corresponding author: XU XY, School of Physical Education, South China Norma University, Guangzhou 510631, China, E-mail: xuxy@scnu.edu.cn

Received April 18, 2018; Accepted April 24, 2018; Published May 03, 2018

Citation: Dong HL, WU HY, Zhao J, Huang YW, Li Z, et al. (2018) Effect of Electrical Stimulation at Different Time on Mitochondrial Function of $\mathrm{C} 2 \mathrm{C} 12$ Myotubes. J Biomol Res Ther 7: 162. doi:10.4172/2167-7956.1000162

Copyright: @ 2018 Dong HL, et al. This is an open-access article distributed under the terms of the Creative Commons Attribution License, which permits unrestricted use, distribution, and reproduction in any medium, provided the original author and source are credited. 
Citation: Dong HL, WU HY, Zhao J, Huang YW, Li Z, et al. (2018) Effect of Electrical Stimulation at Different Time on Mitochondrial Function of C2C12 Myotubes. J Biomol Res Ther 7: 162. doi:10.4172/2167-7956.1000162

Page 2 of 5

\section{Electrical stimulation}

We used custom built electrodes made of $1 \mathrm{~mm}$ platinum wire for electrical stimulation of the $\mathrm{C} 2 \mathrm{C} 12$ myotubes. Two half-circle electrodes were aligned to the side of the well, in order to obtain a maximal area of stimulation. At first, platinum wire electrodes were sterilized with $70 \%$ ethanol and subsequently dried under ultraviolet light. On differentiation day 7, C2C12 myotubes were given one electrical stimulation exposure $(15 \mathrm{~V}, 3 \mathrm{~Hz}, 30 \mathrm{~ms}$, Grass S-48 stimulator). There were four groups: Con (without stimulation); E60 (with stimulation of 60 minutes); E120 (with stimulation of 120 minutes); E180 (with stimulation of 180 minutes).

\section{Determination of MDA, SOD and ROS}

Enzyme assays were performed on cell lysates. MDA, SOD and ROS were measured using commercial assay kits. All metabolite data were normalized to the corresponding cellular protein content. Firstly, MDA (Malondialdehyde) was tested by TBA (Thiobarbituric acid) method. In brief, MDA be condensed with TBA to form a red product, which has a maximum absorption peak at $532 \mathrm{~nm}$ by visible spectrophotometer. Secondly, SOD was tested by NBT method. In brief, under aerobic conditions, azablue tetrazole is reduced to blue methylhydrazone easily, which has a maximum absorption at $560 \mathrm{~nm}$. SOD inhibits the formation of methylhydrazone. So the deeper the blue, the lower will be the enzyme activity. Thirdly, ROS was tested by DCFH-DA method. In brief, DCFH-DA can be changed into DCFH. Both of them have no fluorescence. But ROS can change DCFH into DCF which has fluorescence. The excitation wavelength is $488 \mathrm{~nm}$ and the emission wavelength is $525 \mathrm{~nm}$.

\section{Western blotting}

Briefly, cells were washed with cold phosphate buffer saline (PBS) twice and directly lysed with Laemmli sample buffer, then centrifuged (12000 g, $10 \mathrm{~min}$ ), and the supernatant was removed. The BCA Protein Assay Kit was then used to measure total protein concentration $\left(\mu \mathrm{g} / \mathrm{cm}^{2}\right)$. The left samples were then run on $8-15 \%$ SDS-PAGE, followed by transfer to a nitrocellulose membrane (Bio-Rad-USA). The membrane was blocked for $2 \mathrm{~h}$ at room temperature with $5 \%$ BSA and $0.05 \%$ Tween-20 in phosphate buffer saline. Next, the membrane was incubated with anti-Sirt1, anti- Sirt3, anti- p-ULK (CST), and anti-PGC1 (abcam) overnight at $4^{\circ} \mathrm{C}$. The membrane was washed and incubated with rabbitperoxidase-conjugated secondary antibody at room temperature for $2 \mathrm{~h}$ and the immune-reactive bands were visualized using a chemi-luminescence detection system (Imuno-Star, Bio-Rad). Relative band intensity was quantified using Image J software after normalizing with $\beta$-actin band intensity.

\section{Determination of mitochondrial membrane potential in myotubes by flow cytometry}

TMRM shows fluorescent staining aggregating in mitochondria selectively. Once released to the cell plasma, fluorescence quenching. So TMRM method used to analyze and observe the mitochondrial channel pore activity. In belief, Mitochondria was extracted according to the extraction kit description, and then add a fluorescent probe 100 $\mathrm{nm}$ TMRM to dye the mitochondria in dark for 30 minutes. 4, 11000 $\mathrm{g}$ of centrifugation for 10 minutes. After discarding the supernatant, clean the cock solution twice, 4, centrifuging $11000 \mathrm{~g}, 10$ minutes each time. After discarding the supernatant, the mitochondrial membrane potential was measured by adding the cock solution.

\section{Statistical analysis}

SPSS software, version 16.0, was used to analyse all data presented as means and standard deviation $(\mathrm{M} \pm \mathrm{SD})$. One-way ANOVA and Tukey's post hoc tests were carried out to determine the differences in several time points and two different conditions. Two-way ANOVA were carried out to determine the differences between time and treatment effects. $\mathrm{P}<0.05$ was considered statistically significant.

\section{Results}

\section{Effects of electrical stimulation on myotubes morphology of $\mathrm{C} 2 \mathrm{C} 12$}

C2C12 myotubes that differentiated for 7 days were thick and had the same contractility as in vivo muscles. As can be seen from the data in Figure 1, there were a large number of elongated myotubes. And there were a large number of myotubes with normal structure in the four images. Therefore, there was no significant difference in the morphology of myotubes between different groups.

\section{Effects of electrical stimulation on MDA, ROS and SOD of C2C12}

As can be seen from the data in Table 1, compared with the control group, MDA and ROS increased significantly at $60 \mathrm{~min}$ after electrical stimulation $(\mathrm{p}<0.05)$; MDA and ROS increased significantly at 120 min and 180 min after electrical stimulation $(\mathrm{p}<0.01)$; SOD decreased significantly at $120 \mathrm{~min}(\mathrm{p}<0.05)$ and $180 \mathrm{~min}(\mathrm{p}<0.01)$ after electrical stimulation.

\section{Expression of mechanism protein in $\mathrm{C} 2 \mathrm{C} 12$ myotubes after electrical stimulation}

As can be seen from the data in Figure 2, compared with the control group, the expression of Sirt3, p-ULK and PGC1 increased significantly increased significantly $(\mathrm{p}<0.01)$ and Sirt1 decreased significantly $(\mathrm{p}<0.05)$ at $60 \mathrm{~min}$ of electrical stimulation. The expression of Sirt3 and PGC1 increased significantly at $120 \mathrm{~min}$ of electrical stimulation $(\mathrm{p}<0.01)$. The expression of all proteins did not change significantly at 180 min of electrical stimulation.

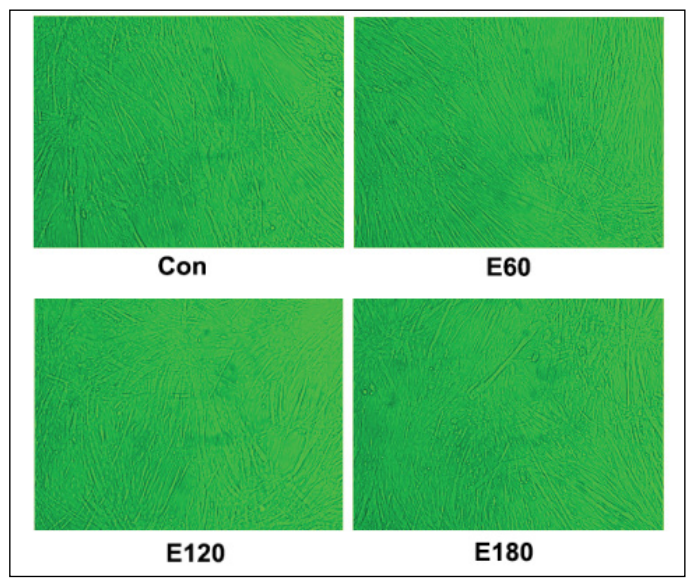

Figure 1: Effect of electrical stimulation on $\mathrm{C} 2 \mathrm{C} 12$ myotubes form.

\begin{tabular}{|c|c|c|c|c|}
\hline & Con & E60 & E120 & E180 \\
\hline MDA $(\mathrm{nmol} / \mathrm{mL})$ & $3.65 \pm 0.14$ & $4.37 \pm 0.21^{*}$ & $4.95 \pm 0.09^{* *}$ & $5.21 \pm 0.15^{\star *}$ \\
\hline SOD (U/mL) & $648.6 \pm 14.7$ & $665.2 \pm 16.0$ & $582.6 \pm 13.7^{*}$ & $550.1 \pm 10.8^{* *}$ \\
\hline ROS (U/mL) & $21.7 \pm 12.4$ & $88.2 \pm 16.7^{*}$ & $155.4 \pm 11.2^{* *}$ & $147.4 \pm 10.9^{* *}$ \\
\hline
\end{tabular}


Citation: Dong HL, WU HY, Zhao J, Huang YW, Li Z, et al. (2018) Effect of Electrical Stimulation at Different Time on Mitochondrial Function of C2C12 Myotubes. J Biomol Res Ther 7: 162. doi:10.4172/2167-7956.1000162

Page 3 of 5

\section{Effect of electrical stimulation on mitochondrial membrane potential of $\mathrm{C} 2 \mathrm{C} 12$ myotubes}

From the data of Figure 3 and Table 2, we can know that compared with the control group, the mitochondrial membrane potential decreased slightly at $60 \mathrm{~min}$ after electrical stimulation ( $p>0.05)$; And the mitochondrial membrane potential decreased significantly at 120 min after electrical stimulation $(\mathrm{p}<0.05)$, and then decreased more significantly at $180 \mathrm{~min}$ after electrical stimulation $(\mathrm{p}<0.01)$.

\section{Discussion}

Reactive oxygen species, the superoxide radicals and its derivatives produced in the electron transport of aerobic respiration, are considered to be the major factors mediating exercise-induced mitochondrial biosynthesis [7]. Loss of balance between the ROS produced by exercise and the body's antioxidant system, results in a series of adaptive stress physiologic responses known as exercise oxidative stress. The level of reactive oxygen species is closely related to exercise. Exercise activates reactive oxygen species quickly, and the recovery period gradually decreased as the movement time continues to accelerate [8]. Compared with the quiet state, the production and physiological effects of reactive oxygen species are very different during exercise. In resting state, the level of reactive oxygen species is generally very low, and the damage to mitochondrial function is small. During exercise, the level of reactive oxygen species, oxidative stress and cell injury increase. Excessive reactive oxygen species stimulates mitochondrial lipid peroxidation and produces more malondialdehyde. Malondialdehyde crosslinks with mitochondrial membrane lipids to form (schiff) enamine base, which affects mitochondrial electron transport and function [9].

A

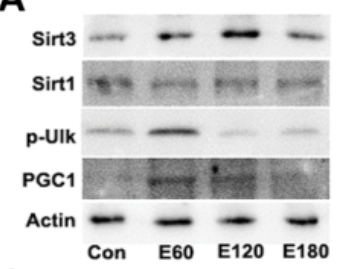

C

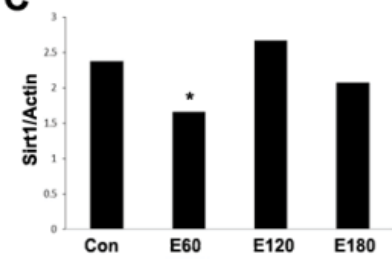

E

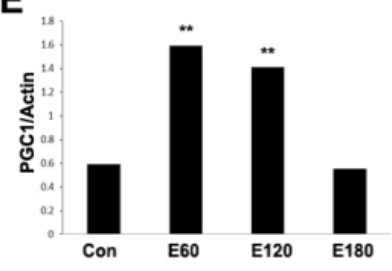

${ }^{*} p<0.05 ;{ }^{* *} p<0.01$

Figure 2: Effect of electrical stimulation on mechanism proteins of $\mathrm{C} 2 \mathrm{C} 12$ myotubes.

\begin{tabular}{|c|c|c|c|c|}
\hline & Con & E60 & E120 & E180 \\
\hline TMRM & $11.13 \pm 0.13$ & $9.87 \pm 0.09$ & $7.32 \pm 0.08^{*}$ & $6.57 \pm 0.11^{* *}$ \\
\hline${ }^{*} \mathbf{p}<\mathbf{0 . 0 5} ;{ }^{* *} \mathbf{p}<\mathbf{0 . 0 1}$ & &
\end{tabular}

Table 2: Effect of electrical stimulation on TMRM of C2C12 myotubes.
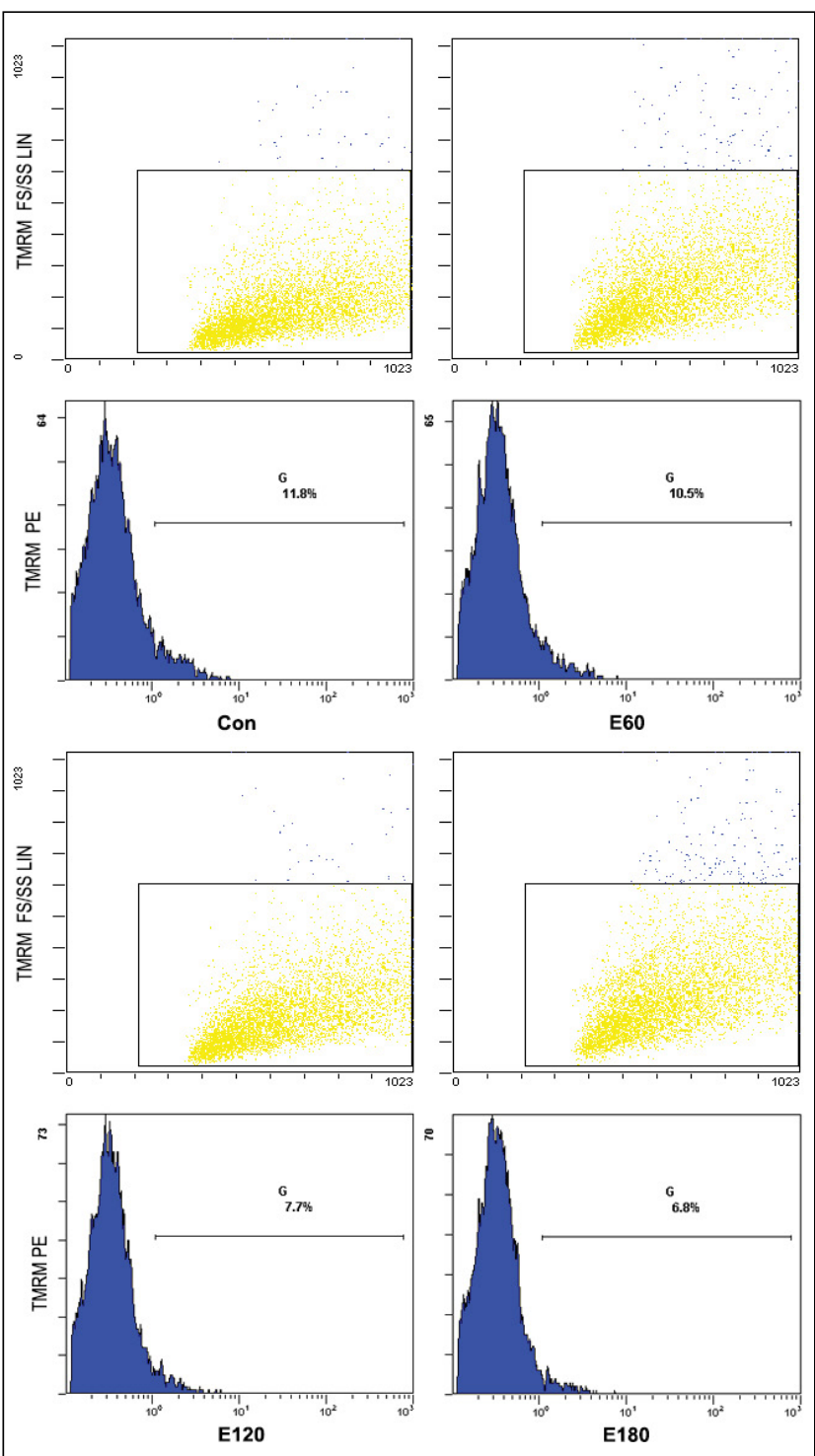

Figure 3: Results of TMRM of $\mathrm{C} 2 \mathrm{C} 12$ myotubes after electrical stimulation.

However, transient reactive oxygen species caused by exercise also stimulate mitochondrial biosynthesis [7]. Therefore, moderate reactive oxygen species is necessary to maintain mitochondrial function.

Mitochondria are the main source of reactive oxygen species, and also are first attacked by reactive oxygen species. Reactive oxygen species attack mitochondria, leading to mitochondrial dysfunction. Mitochondrial membrane potential is the potential difference between ions in the mitochondrial membrane and ions outside the membrane. It is the basic condition for mitochondrial oxidative phosphorylation and proton transmembrane transport, reflecting mitochondrial function. The decrease of mitochondrial membrane potential is the precursor of cell oxidative damage. In myocardial aging model, we can observe a series of mitochondrial function decrease, such as decrease in mitochondrial membrane potential, increase in proton leakage and increase in reactive oxygen species production [10]. Skeletal muscle is the most important part of energy metabolism. So it is very important to maintain the integrity of structure and function in skeletal muscle mitochondrial. The increase of skeletal muscle degradation and 
apoptosis were related to increased reactive oxygen species, changes in mitochondrial membrane potential and DNA oxidative damage. The mitochondrial structure and function are closely related to exercise ability. Any changes of mitochondria will directly or indirectly affect skeletal muscle function. Exercise is also an important factor affecting the structure and function of skeletal muscle mitochondria. Exercise directly or indirectly interferes with mitochondrial biosynthesis and function through transcription and translation [11]. It found that aerobic exercise is beneficial to maintain the mitochondrial membrane potential of myocardial cells in aging rats in a 12 weeks aerobic exercise study [12].

It is well known that different exercise causes different phenotype changes in cells and tissues. The endurance training is characterized by high oxygen intake, low shrinkage and mitochondrial dependence. Therefore, endurance training improves the oxidative ability and mitochondrial function of skeletal muscle [13]. In this study, it was found that, as the time of electrical stimulation increased, there was no significant change in the shape of the myotubes, but MDA and ROS gradually increased, SOD and mitochondrial membrane potential gradually decreased. The results showed that the electrical stimulation intensity of this experiment simulated aerobic exercise and successfully resulted in oxidative stress and decreased mitochondrial function. However, the changes of motor oxidative stress and mitochondrial function caused by different electrical stimulation are different, so the mechanism needs to be studied.

The Sirts are NAD+ dependent deacetylase, which regulates multiple cellular processes including metabolic balance. Sirt1 is a redox sensor mainly located in the nucleus. Sirt1 is a major regulator of mitochondrial biosynthesis in skeletal muscle, and regulates the mitochondrial function mainly through deacetylation and PGC1 activation [14-16]. NADH affects downstream substrates such as PGC1 by inhibiting Sirtl activity, thus regulating mitochondrial biological function [17]. Cell experiments after $\mathrm{H}_{2} \mathrm{O}_{2}$ revealed that, cell overoxidation induced phosphorylation of c-JNK, leading to proteinase degradation of Sirt1 [18]. Similarly, compared to aerobic exercise, acute hypoxia induced more intense oxidative stress in vivo, leading to more severe oxidative damage, and decreased Sitr1 and NAD/NADH ratios [19-21]. However, some studies have shown that the deacetylation activity of Sirt1 is not required to maintain mitochondrial function during exercise [22]. Sirt1 has three phosphorylation sites, including Ser27, Ser47 and Thr530. Phosphorylation of Ser27 and Thr530 enhances enzyme activity and nuclear location, while phosphorylation at Ser47 inhibits the deacetylase activity of Sirt1 [23]. In this study, the expression of Sirt1 decreased after 1 hour electric stimulation, but unchanged after 2 and 3 hours. The reason for the result may be that acute hypoxia induced by 1 hour electrical stimulation causes oxidative stress, which leads to degradation of Sirt1. However, there was no change in Sirt 1 after 2 and 3 hours electrical stimulation, which also confirmed by previous studies, suggesting that Sirt1 was not need to maintain mitochondrial function.

Sirt3 is a mitochondrial protein that regulates energy balance, oxidative metabolism, oxidative stress and cell damage [24]. Reduced Sirt3 leads to mitochondrial function changes, elevated oxidative stress, activation of JNK, and impaired insulin signals. Although Sirt 3 widely targets many substrates as a mitochondrial deacetylase, the exact molecular mechanism about its effect on the oxidative metabolism in skeletal muscle remains unclear [25]. Previous studies have found that PGC1 regulates the activity of Sirt 3 as an endogenous stimulant in both skeletal and hepatocytes [26]. Under different pathological and physiological conditions, Sirt3 controls mitochondrial oxidation and ROS production, and activates ROS scavenging enzyme [2729]. Therefore, it is important to study the affection of Sirt3 on mitochondrial oxidation function and energy balance. In addition, McGee et al. suggested that PGC1 and p-ULK regulated skeletal muscle volume and muscle fiber type [30]. Both Sirt3 and Sirt1 can promote mitochondrial biosynthesis and fatty acid oxidation through PGC1, but in different ways. Sirt3 activates PGC1, and Sirt1 promotes deacetylation of PGC1 [14]. In this study, Sirt3 increased after 1 and 2 hours electrical stimulation. The reason for the result may be that the free radical produced by electrical stimulation mainly comes from mitochondria, which are also the first to be attacked. While expressed Sirt3 mainly in mitochondria and therefore in higher levels after electrical stimulation. Although it has been found that exercise regulate the expression of Sirt3 instead of Sirt1 in the skeletal muscle [31]. But in different environments and organizations, about the work about the collaborative of the two Sirts deserves further study.

This study found that the expression of Sirt3 increased after 1 and 2 hour electrical stimulation, while Sirt1 decreased after 1 hour electrical stimulation, suggesting that the exercise intensity of this experiment does maintain mitochondrial function of skeletal muscle through Sirt3 rather than Sirt1. In addition, compared with control group, P-ULK and PGC1 increased significantly after 1 hour electrical stimulation. PGC1 increased and mitochondrial membrane potential decreased significantly after 2 and 3 hour electrical stimulation. The results showed that moderate exercise could activate oxidative stress, promote the expression of Sirt3, PGC1, p-ULK, and the recovery of mitochondrial membrane potential. While excess movement activates the opposite. In the following study, SiRNA interference will be used to observe the role of Sirt3, PGC1 and p-ULK in the mitochondrial function of skeletal muscle, and to more precisely prove the mechanism of oxidative stress affecting mitochondrial function of skeletal muscle.

\section{Conclusion}

Moderate time electrical stimulation (60 and 120 mins) could activate oxidative stress, promote the expression of Sirt3, PGC1, p-ULK, and then promote mitochondrial membrane potential recovery. While excessive time electrical stimulation (180 mins) had the opposite effect. In addition, the changes of mitochondrial membrane potential induced by electrical stimulation were mainly controlled by Sirt3 rather than Sirt1.

\section{Funding}

Guangdong Natural Science Foundation (2017A030310178); Educational and Teaching Reform Subject in Universities of China (82617058).

\section{References}

1. Menshikova EV, Ritov VB, Ferrell RE, Azuma K, Goodpaster BH, et al. (2007) Characteristics of skeletal muscle mitochondrial biogenesis induced by moderate-intensity exercise and weight loss in obesity. J Appl Physiol 103: $21-27$

2. Goldman SJ, Taylor R, Zhang Y, Jin S (2010) Autophagy and the degradation of mitochondria. Mitochondrion 10: 309-15.

3. Lo VF, Carnio S, Vainshtein A, Sandri M (2014) Autophagy is not required to sustain exercise and PRKAA1/AMPK activity but is important to prevent mitochondrial damage during physical activity. Autophagy 10: 1883-94.

4. Shi L, Zhang T, Zhou Y, Zeng X, Ran L, et al. (2015) Dihydromyricetin improves skeletal muscle insulin sensitivity by inducing autophagy via the AMPK-PGC1a-Sirt3 signaling pathway. Endocrine 50: 378-389.

5. Brook MS, Wilkinson DJ, Mitchell WK, Lund JN, Szewczyk NJ, et al. (2015) Skeletal muscle hypertrophy adaptations predominate in the early stages of resistance exercise training, matching deuterium oxide-derived measures of muscle protein synthesis and mechanistic target of rapamycin complex signaling. FASEB J 29: 4485-4496. 
Citation: Dong HL, WU HY, Zhao J, Huang YW, Li Z, et al. (2018) Effect of Electrical Stimulation at Different Time on Mitochondrial Function of C2C12 Myotubes. J Biomol Res Ther 7: 162. doi:10.4172/2167-7956.1000162

Page 5 of 5

6. Irving M (2017) Regulation of Contraction by the Thick Filaments in Skeletal Muscle. Biophys J 113: 2579-2594.

7. Gomez-Cabrera MC, Domenech E, Romagnoli M, Arduini A, Borras C, et al. (2008) Oral administration of vitamin C decreases muscle mitochondria biogenesis and hampers training-induced adaptations in endurance performance. Am J Clin Nutr 87: 142-149.

8. Bo H, Jiang N, Ma G, Qu J, Zhang G, et al. (2008) Regulation of mitochondrial uncoupling respiration during exercise in rat heart: role of reactive oxygen species (ROS) and uncoupling protein 2. Free Radic Biol Med 44: 1373-1381.

9. Lee SH, Nam BY, Kang EW, Han SH, Li JJ, et al. (2010) Effects of an ora adsorbent on oxidative stress and fibronectin expression in experimental diabetic nephropathy. Nephrol Dial Transplant 25: 2134-2141.

10. Savitha S, Panneerselvam C (2006) Mitochondrial membrane damage during aging process in rat heart: potential efficacy of L-carnitine and $D L$ alpha lipoic acid. Mech Ageing Dev 127: 349-55

11. Russell AP, Foletta VC, Snow RJ, Wadley GD (2014) Skeletal muscle mitochondria: a major player in exercise, health and disease. Biochim Biophys Acta 1840: 1276-84

12. Zoladz JA, Koziel A, Broniarek I, Woyda-Ploszczyca AM, Ogrodna K, et al (2017) Effect of temperature on fatty acid metabolism in skeletal muscle mitochondria of untrained and endurance-trained rats. PLoS One 12: e0189456.

13. Saleem A, Adhihetty PJ, Hood DA (2009) Role of p53 in mitochondrial biogenesis and apoptosis in skeletal muscle. Physiol Genomics 37: 58-66.

14. Gurd BJ (2011) Deacetylation of PGC-1a by SIRT1: importance for skeletal muscle function and exercise-induced mitochondrial biogenesis. Appl Physiol Nutr Metab 36: 589-597.

15. Zhu L, Wang L, Cao F, Liu P, Bao H, et al (2018) Modulation of transport and metabolism of bile acids and bilirubin by chlorogenic acid against hepatotoxicity and cholestasis in bile duct ligation rats: involvement of SIRT1-mediated deacetylation of FXR and PGC-1 $\alpha$. J Hepatobiliary Pancreat Sci 25: 195-205.

16. Gerhart-Hines Z, Rodgers JT, Bare O, Lerin C, Kim SH, et al. (2007) Metabolic control of muscle mitochondrial function and fatty acid oxidation through SIRT1/ PGC-1alpha. EMBO J 26: 1913-1923.

17. Lagouge M, Argmann C, Gerhart-Hines Z, Meziane H, Lerin C, et al. (2006) Resveratrol improves mitochondrial function and protects against metabolic disease by activating SIRT1 and PGC-1alpha. Cell 127: 1109-1122.

18. Gao Z, Zhang J, Kheterpal I, Kennedy N, Davis RJ, et al. (2011) Sirtuin 1 (SIRT1) protein degradation in response to persistent c-Jun $\mathrm{N}$-terminal kinase 1 (JNK1) activation contributes to hepatic steatosis in obesity. J Biol Chem 286 : 22227-22234.
9. Morales-Alamo D, Ponce-González JG, Guadalupe-Grau A, Rodriguez-Garcia L, Santana A, et al. (2012) Increased oxidative stress and anaerobic energy release, but blunted Thr172-AMPKa phosphorylation, in response to sprin exercise in severe acute hypoxia in humans. J Appl Physiol 113: 917-928.

20. Morales-Alamo D, Calbet JA (2014) Free radicals and sprint exercise in humans. Free Radic Res 48: 30-42.

21. Place N, Ivarsson N, Venckunas T, Neyroud D, Brazaitis M, et al. (2015) Ryanodine receptor fragmentation and sarcoplasmic reticulum Ca2+ leak after one session of high-intensity interval exercise. Proc Natl Acad Sci 112: 15492 15497.

22. Menzies KJ, Chabi B, Hood DA, Schenk S, Philp A, et al. (2012) Commentaries on viewpoint: does SIRT1 determine exercise-induced skeletal muscle mitochondrial biogenesis: differences between in vitro and in vivo experiments J Appl Physiol 112: 929-930.

23. Lojewski X, Srimasorn S, Rauh J, Francke S, Wobus M, et al. (2015) Perivascular Mesenchymal Stem Cells From the Adult Human Brain Harbor No Instrinsic Neuroectodermal but High Mesodermal Differentiation Potential. Stem Cells Transl Med 4: 1223-1233.

24. Wang X, Tang H, Chen Y, Chi B, Wang S, et al. (2016) Overexpression of SIRT3 disrupts mitochondrial proteostasis and cell cycle progression. Protein Cell 7: 295-299.

25. Lombard DB, Alt FW, Cheng HL, Bunkenborg J, Streeper RS, et al. (2007) Mammalian Sir2 homolog SIRT3 regulates global mitochondrial lysine acetylation. Mol Cell Biol 27: 8807-8814.

26. Park SJ, Ahmad F, Philp A, Baar K, Williams T, et al. (2012) Resveratrol ameliorates aging-related metabolic phenotypes by inhibiting cAMP phosphodiesterases. Cell 148: 421-33.

27. Giralt A, Villarroya $F$ (2012) SIRT3, a pivotal actor in mitochondrial functions: metabolism, cell death and aging. Biochem $\mathrm{J}$ 444: 1-10.

28. Liu J, Li D, Zhang T, Tong Q, Ye RD, et al. (2017) SIRT3 protects hepatocytes from oxidative injury by enhancing ROS scavenging and mitochondrial integrity. Cell Death Dis 8: e3158.

29. Zeng L, Yang Y, Hu Y, Sun Y, Du Z, et al. (2014) Age-related decrease in the mitochondrial sirtuin deacetylase Sirt3 expression associated with ROS accumulation in the auditory cortex of the mimetic aging rat model. Plos One 9: e88019.

30. McGee SL, Hargreaves M (2010) AMPK-mediated regulation of transcription in skeletal muscle. Clin Sci 118: 507-18.

31. Palacios OM, Carmona JJ, Michan S, Chen KY, Manabe Y, et al. (2009) Diet and exercise signals regulate SIRT3 and activate AMPK and PGC-1alpha in skeletal muscle. Aging (Albany NY) 1: 771-783. 